\title{
Electroless or Autocatalytic Coating of Microparticles for Laser Fusion Targets
}

by

Anton Mayer

Duane S. Catlett

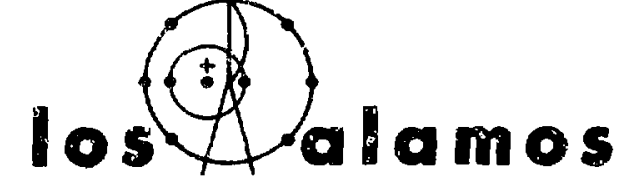

sciemsitic laboratory

of the University of Californio

los alAMOS, NeW MEXICO 87545<smiles>[13CH3][13CH3]</smiles>

An Affirmative Action/Equal Opportunity Employer 
Work supported by the US Energy Research and Development Administration, Division of Laser Fusion.

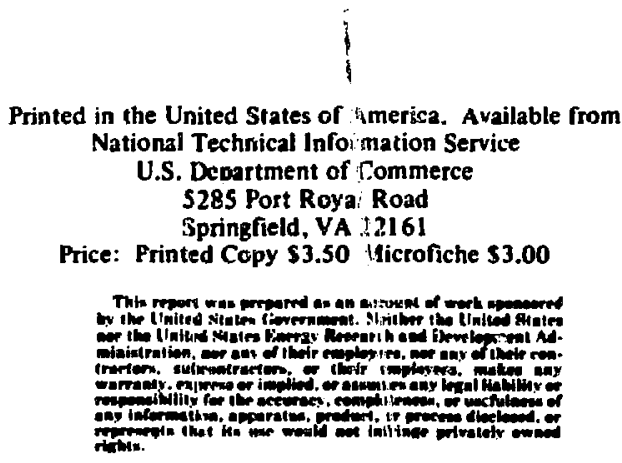




\title{
ELECTROLESS OR AUTOCATALYTIC COATING OF MICROPARTICLES FOR LASER FUSION TARGETS
}

by

Anton Mayer and Duane S. Catlett

\begin{abstract}
Use of a novel device for applying uniform metallis, coatinge to opherical microparticles is deseribed. The apparatus deponits electroless metal coatings on hollow, thin-vialled metal or wensitized nonmethllic miaromandrels. The apparatun aud process were developed for fabrication of microsphere prensure vessels for use as targets in laser-initiated fusion research.
\end{abstract}

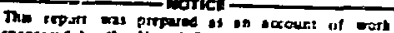

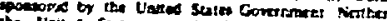

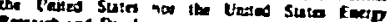

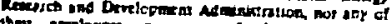

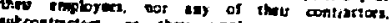

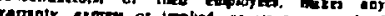

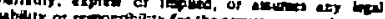

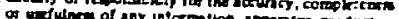

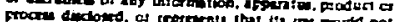
teinup prinidy owedichis.

\section{INTRODUCTION}

In research on laser-initiated pure fusion, target design has been limited severely by the lack of sufficiently energetic lasens. The most difficult constraint is that of size. Present laser fusion targets range from $50 \mathrm{tn} 500 \mu \mathrm{m}$ in diameter.

Figure I shows a hypothetical target.' The inner shell is generally obtained from a commercial source, and in many designs it is considered to be an inert layer, whose sole purpose is to serve as a mandrel upon which to deposit the design layers. Regardless of the coating technique, during the deposition process, the microparticles must be forced to move individually and randomly in the coating medium to ensure wall thickness uniformity and homoganeity of the deposit.

Several techniques for applying metal shell b to microspheres ${ }^{i t}$ have been evaluated. They include physical vapor deposition (PVD), chemical vapor deposition (CVD), sputter deposition, and electroand electroless plating. An apparatus developed to electroplate microspheres for laser fusion targets has been reported." Here, we describe an apparatus developed to electrolessly plate such microspheres.

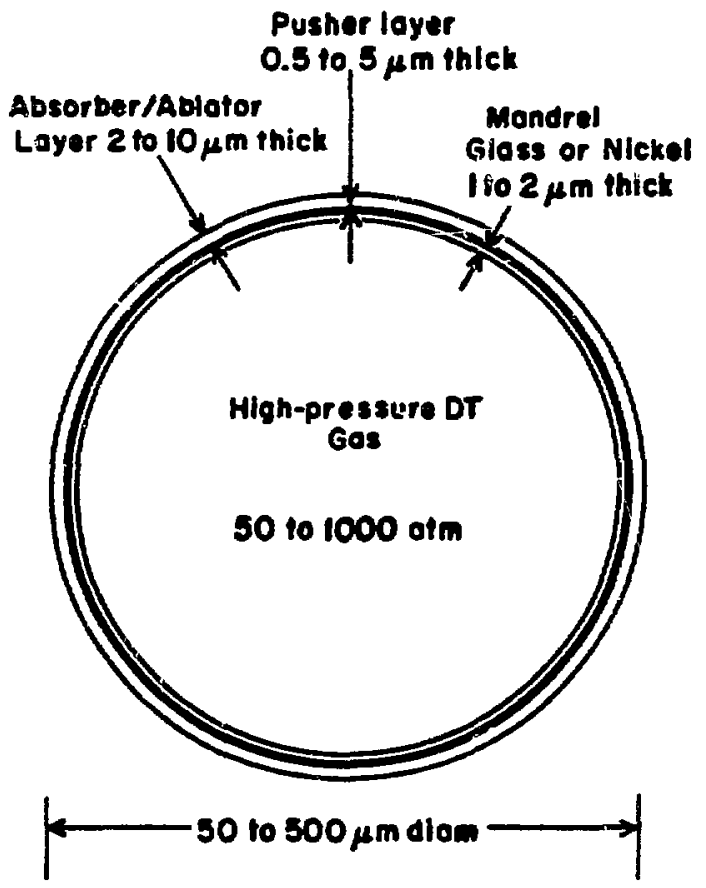

Fig. 1.

A hypothetical structured, multilayered laser fusion terget. 


\section{APPARATUS}

Electrole58, or autocatalytic, plating has extensive decorative and engineering applications in the metal-finishing induatry. Lagge parts are plated on racks; small parts are efficiently processed in bulk in screen baskets or perforated barrels. Applying thick, uniform coatings to hollow micropaticles presented a challenge, because existing techniques could not be applied directly.

The initial approuch to coating thin-walled metal or metal-coated glass shells, at both LASL and Bendix Kanses City, was to suspend the particles in the solution vortex or in a vigorous!y stirred electroless plating solution until the desired coating thickness was obtained. In theory, the particles would ride in the solution vortex and thus be plated uniformly. In practice, however, some particles were drawn into the solution meniscus where their motion and the deposition essentially ceased. As the plating progressed, other particles became denser than the solution medium, sank to the bottors of the plating tank, and agglomerated. A new opparatus makes it practical to electrolessly piatz discrete micropartj. cles with uniformly thick deposits.

The electroless plating apparatus is shown in Fig. 2. The microperticles are confined in a cylindrical reaction chember by fine mesh screens at top and bottom. The cylinder wall extends beyond both screens. The top extension serves as a solution overnow and irtake cavity. The bottom extension is fitted over a stationary piston. The plating apparatus is immersed vertically in the electroless plating solution, and the extension rod is attached to a mechanical or pneumatic direction-reversing drive mechanism. The reaction chamber is forced intermittently up and down over the atationary piston. The 'irection-reversing cycle time is variable from 1 to $10 \mathrm{~s}$. The moving chamber's stroke length is adjusted to purge the reaction chamber with fresh solution continually.

The solution movement through the reaction chamber moves the particles randomly during metal deposition. When the motion is downward, plating solution forced through the bottom screen into the reaction chamber lifts and disperses the particles that are denser than the solution. When the motion is upward, solution drawn into the cavity by auction through the top screen disperses the particles that

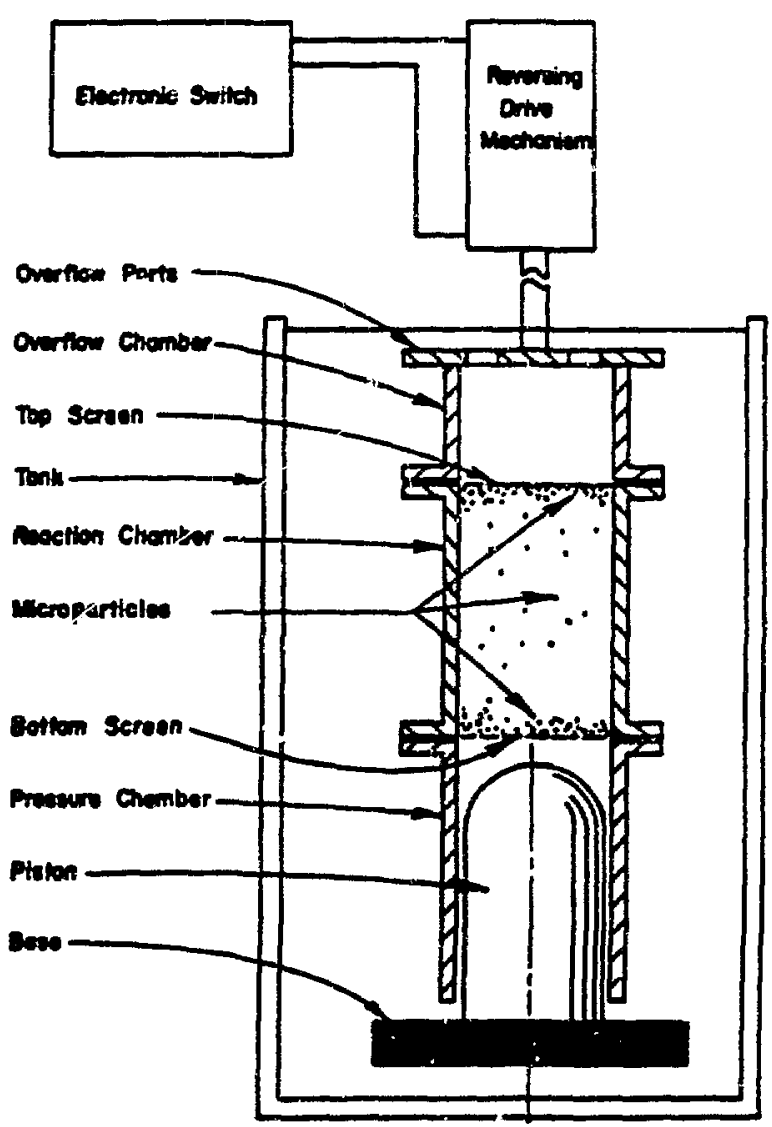

Fig. 2.

An apparatus for autocatalytic plating of dis. crete microparticies.

float. Evolved hydrogen, which normally accompanies electmleas deponition, is forced out of the reaction chamber during the downward stroke, thereby keeping the cavity filled with plating solution.

\section{RFSURT}

Uniform, fully dense deposits with excellent sur-

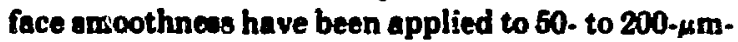
diam opherical mandrels. The agglomeration inherent in previous techniques has been virtually eliminated. Metal distribution from particle to particle within the same batch is excellent. 
Figure 3, a metallurgical cross section of an experimental laser-fusion target, shows a uniform coating of $16 \mu \mathrm{m}$ of electroless nickel over the thinwalled $(2-\mu \mathrm{m})$ spherical glass mandrel.

Figure 4 is a scanning electron micrograph of an electroless nickel coated laser target. The surface

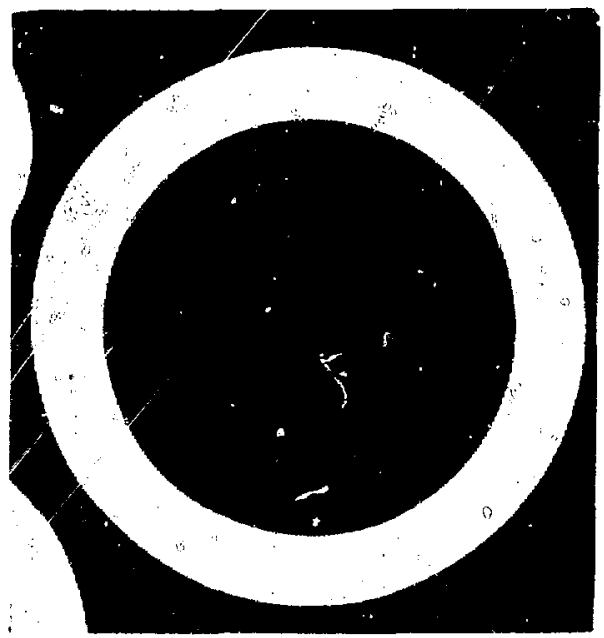

Fig. 3.

Metallographic cross section of $16 \mu \mathrm{m}$ of electroless nickel plated on a thin-walled glass mandrel.

(500X)

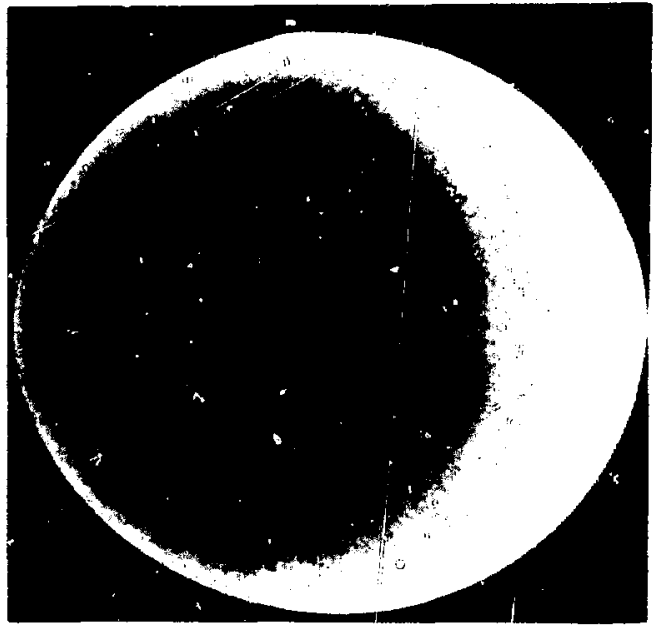

Fig. 4.

Scanning electron micrograph of an electroless nicket-ingted luser target.

(550X) finish of particles coated in this apparatus is as good as, or better than, that of the starting substrate.

We have thus far applied electroless nicke! coatings to both metal and metallized-glass spheres, 2 to $22 \mu \mathrm{m}$ thick. Proprietary baths from the Shipley Company and the Sel Rex Corporation were used as plating solutions. Exceedingly fine-grained, smooth coatings have been deposited from the Shipley "Niculoy 22" bath and the Sel Rex "Lectroless $\mathrm{Ni}$ " process. The Shipley bath deposits a nickelphosphorous-copper alloy that is typically about 12 $w t \% \mathrm{P}, 1 \mathrm{wt} \% \mathrm{Cu}$, and $87 \mathrm{wt} \% \mathrm{Ni}$. The Sel Rex solution deposits a nickel-phosphorous alloy whose phosphorous content is $<2 \mathrm{wt} \%$. Batch sizes range from only a few spheres to over 100000 .

\section{SUMMARY}

We have developed a method and apparatus for applying very uniform coatings to discrete microparticles by electroless techniques. Agglomeration or bridging of the particles is prevented by moving them randomly during deposition. The microparticle density is not critical and may be less tran, equal to, or more than that of the plating solution. Although all particles coated thus far have been hollow spheres, the method is applicable ts discrete irregular microparticles.

\section{ACKNOWLEDGMENTS}

Grateful acknowledgments are extended to C. A. Javorsky and J. R. Bradberry, who were responsible for the metallography, and to R. R. Bryant, for fabricating the apparatus.

\section{REFEIRENCES}

1. R. J. Fries and E. H. Farnum, "Laser Fusion Target. Fabrication." Los Alamos Scientific Laboratory report LA-5703-Rev. (1974).

2. J. W. Havenhill, "I973 Laser Target Activities," Los Alamos Scientific Laboratory unpublished data. 
3. W. J. McCreary, "Microspherical Laser Targets by CVD," Proc. Conf. Chemical Vapor Deposition, Electrochemical Society, Princeton, New Jersey (1975), p. 714.

4. Gary A. Simonsic and Billy W. Powell, "Vapor Phase Coatings of Metals and Organics for Laser Fusion Target Applications," Los Alamos Scientific Laboratory unpublished data.
5. Anton Mayer and Duane S. Catiett, "Electrolytic Coating of Microparticles for Laser Fusion Targets," Los Alamos Scientific Laboratory report LA-6584 (1976).

6. Anton Mayer, U.S. Patent Application No. SN 613849 